\title{
A Comparative Study on Internal Fixation Using Long Proximal Intramedullary Nail for the Treatment of Humeral Shaft Fracture according to Fracture Types
}

\author{
Chang-Hyuk Choi, Chung-Mu Jun, Jun-Young Kim ${ }^{\square}$ \\ Department of Orthopaedic Surgery, Daegu Catholic University College of Medicine, Daegu, Korea
}

Background: This study was conducted to compare the radiological and clinical outcomes of internal fixation using a Polarus humeral nail for treatment of a humeral shaft fracture according to fracture types.

Methods: From 43 patients, 13 were excluded and 30 patients were included. The 30 patients were divided into 2 groups: 15 in group I (Orthopaedic Trauma Association/Arbeitsgemeinschaft für Osteosynthesefragen classification type A and B) and 15 in group II (type C). The mean age was 63.1 years (range, 20-87 years), and mean follow-up period was 2.3 years (range, 1.0-6.1 years). The causes of injuries were as follows: 12, traffic accidents; 14 , simple slips; 2, simple falls; 2, contusions after lower energy trauma. Radiological and clinical evaluations were performed.

Results: Radiological union was confirmed by plain anteroposterior and lateral radiographs on average of 5.0 months in group I, and 8.4 months in group II, respectively. Differences between the two groups were statistically significant $(p<0.01)$. The clinical union value was 1.6 in group I, and 2.0 months in group II, but these values did not differ significantly $(p=0.441)$. The mean Korean shoulder scoring system scores were 89.7 and 90.6, which did not differ significantly $(p=0.352)$.

Conclusions: Intramedullary nailing using the Polarus humeral nail is considered to be a good treatment modality for all types of humeral shaft fractures. Additionally, the Polarus humeral nail can be an optimal choice for the treatment of complex type fractures such as segmental or comminuted humeral shaft fractures.

(Clin Shoulder Elbow 2019;22(2):87-92)

Key Words: Humeral shaft fracture; Polarus humeral nail; Radiological and clinical union

\section{Introduction}

Humeral fractures account for approximately $5 \%$ to $8 \%$ of all extreme fractures, and humeral shaft fractures comprise about $3 \%$ of these. ${ }^{1,2)}$ Most humeral shaft fractures can be managed with non-operative treatments because they are undisplaced or minimally displaced. ${ }^{2,3)}$ In addition, conservative treatment of humeral shaft fractures can be applied in most patients because the range of motion of the shoulder and elbow is large, and functional impairment is rare, even if there is a shortening.
However, although the results of non-operative treatment are satisfactory, various surgical devices such as external fixation, plates, intramedullary nails and multiple pins for humeral shaft fractures have been applied, and surgical indications are gradually expanding to less complex fractures. Among the several surgical options, intramedullary nails have been considered less invasive because they require less extensive soft tissue disability than plates. ${ }^{4}$

Originally, the Polarus intramedullary nail (Acumed LLC, Hillsboro, OR, USA) was developed as a specialized device to treat

Received September 2, 2018. Revised April 14, 2019. Accepted April 21, 2019.

Correspondence to: Jun-Young Kim

Department of Orthopaedic Surgery, Daegu Catholic University College of Medicine, 33 Duryugongwon-ro 17-gil, Nam-gu, Daegu 42472, Korea

Tel: +82-53-621-4487, E-mail: dr.junyoung@gmail.com, ORCID: https://orcid.org/0000-0003-4700-3041

IRB approval: Daegu Catholic University College of Medicine (No. CR-18-128-L).

Financial support: None. Conflict of interests: None. 
proximal humeral fractures by allowing screw stabilization in the humeral head and tuberosities. ${ }^{5)}$ However, we have used this device to treat various types of humeral shaft fractures according to the Orthopaedic Trauma Association (OTA)/Arbeitsgemeinschaft für Osteosynthesefragen $(\mathrm{AO})$ combined classification system. Although several studies have been conducted to treat humeral shaft fractures using the Polarus intramedullary nail, few have compared the radiological and clinical results according to fracture types. Therefore, this study was conducted to compare the radiological and clinical outcomes of internal fixation using the Polarus humeral nail for the treatment of humeral shaft fracture according to the OTA/AO fracture classification system.

\section{Methods}

\section{Demographic Data}

Between December 2005 and April 2018, one surgeon $(\mathrm{CHC})$ performed internal fixation using a Polarus intramedullary nail over $220 \mathrm{~mm}$ in length for treatment of humeral shaft fractures in 43 patients. Of these 43 patients, 13 were excluded for the following reasons: death before 1-year of follow-up (3 patients), follow-up loss (4 patients), pathologic fractures (2 patients), and use of other intramedullary nails (4 patients). Finally, 30 patients (12 males and 18 females) were included in this study. All fractures were classified based on the OTA/AO classification system, and the 3 main fracture types were divided into simple (type A, Fig. 1), wedge (type B, Fig. 2), and complex fractures (type $C$, Fig. 3). Fracture patterns included 12 type A, 3 type $\mathrm{B}$, and 15 type $\mathrm{C}$. The 30 patients were divided into two groups: 15 in group I (type A and B) and 15 in group II (type C) (Table 1). Type A and B were classified into one group, and type $\mathrm{C}$ were categorized into another group according to the difficulty making cortical bone contact when the intramedullary nail was inserted. The mechanisms of initial injury included 12 traffic accidents, 14 simple slips, 2 simple falls, and 2 contusions after lower energy trauma. The accompanying injuries were mostly accompanied by traffic accidents, and there were two cases of neurosurgical problems caused by multiple fractures. The mean duration time from initial injury to surgical treatment was 4.7 days; however, it was only 2.8 days when three
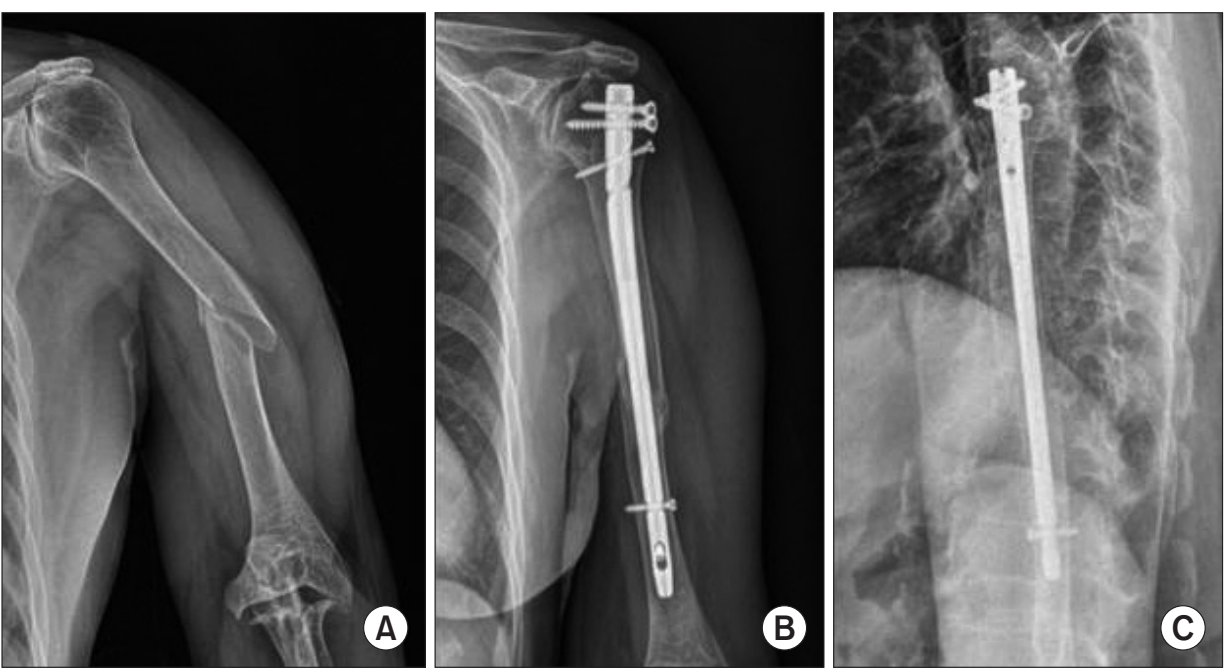

Fig. 1. Simple radiographs of type A fracture. Preoperative (A) and 4 months of follow-up (B) anteroposterior radiographs. (C) Lateral radiograph at the 4 months of follow-up.
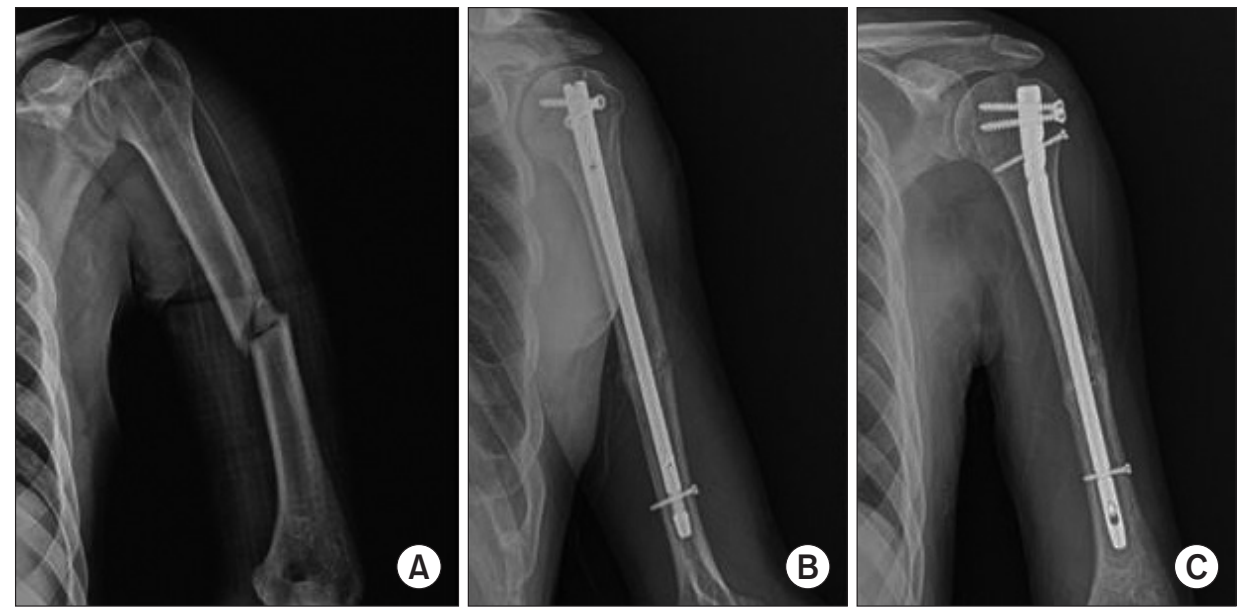

Fig. 2. Simple radiographs of type B fracture. Preoperative (A) and 5 months of follow-up (B) anteroposterior radiographs. (C) Lateral radiograph at the 5 months of follow-up. 

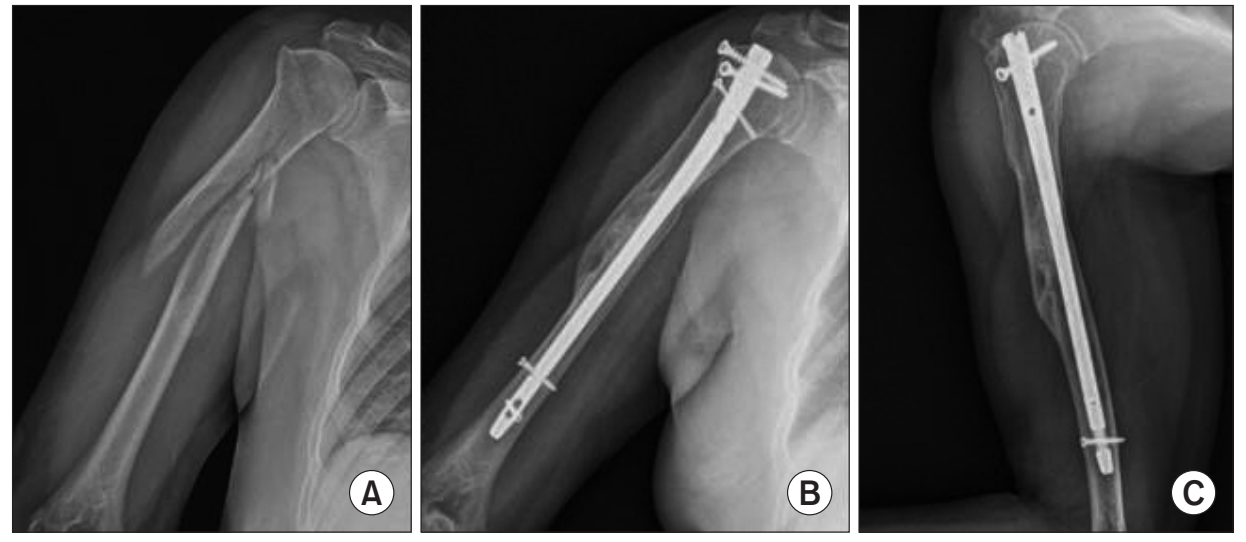

Fig. 3. Simple radiographs of type $C$ fracture. Preoperative (A) and 1 year of followup (B) anteroposterior radiographs. (C) Lateral radiograph at the 1 year of follow-up.

Table 1. Patients' Demographics

\begin{tabular}{lcc}
\hline \multicolumn{1}{c}{ Variable } & Group I $(\mathrm{n}=15)^{\dagger}$ & Group II $(\mathrm{n}=15)^{\dagger}$ \\
\hline Mean age at the time of surgery $(\mathrm{yr})$ & 63.7 & 62.5 \\
Sex (male/female) & $4 / 11$ & $8 / 7$ \\
Injured side (right/left) & $6 / 9$ & $7 / 8$ \\
\hline Injury mechanism (t/s/f/c) & $9 / 5 / 0 / 1$ & $3 / 9 / 2 / 1$ \\
\hline Fracture type (A/B/C) & $12 / 3 / 0$ & $0 / 0 / 15$ \\
Mean follow-up period (yr) & 2.0 & 2.7 \\
\hline
\end{tabular}

Values are presented as mean or number only.

t: traffic accident, s: simple slip, f: simple fall, c: contusion after lower energy trauma.

*Types were based on the Orthopaedic Trauma Association (OTA)/Arbeitsgemeinschaft für Osteosynthesefragen $(\mathrm{AO})$ classification system, and the 3 main fracture types were divided into simple (type A), wedge (type B), and complex fractures (type C).

${ }^{\dagger}$ Group I: type A and B, Group II: type C according to the AO/OTA fracture classification system.

patients who needed other medical procedures due to multiple fractures and one patient who failed in non-operative treatment were excluded. The mean age was 63.1 years (range, 20-87 years), and the mean follow-up period was 2.3 years (range, 1.0-6.1 years). Surgery was performed with closed reduction and internal fixation in all patients, and no open reduction was conducted. The radiological union was defined as when the callus was clearly observed in more than $50 \%$ of the fracture lines on simple anteroposterior (AP) and lateral radiographs. Evaluation of the radiological union was performed by two orthopedic surgeons ( $\mathrm{CHC}$ and $\mathrm{JYK})$, and the consensus was determined by discussion if the results of the evaluation were different. The clinical union was defined as the absence of postoperative pain, tenderness, and false motion on the fracture site. The degree of final functional recovery was measured using the Korean shoulder scoring system (KSS). Complications including radial nerve palsy, subacromial impingement, and migration of the proximal cancellous screw were examined. Radial nerve palsy was defined as wrist and thumb extension of motor grade 4 or less, and subacromial impingement was defined as a case of pain because of nail protrusion with glenohumeral joint movement. Migration of the proximal cancellous screw was determined as when the head of the screw was pulled out more than $3 \mathrm{~mm}$ from the articular margin of humeral head.

\section{Surgical Procedures}

Surgery was performed in a beach chair position under general anesthesia for all cases. As a surgical approach for intramedullary nail insertion, a 3 to $5 \mathrm{~cm}$ vertical skin incision was performed on the lateral margin of acromion, and then extended parallel to the deltoid muscle fibers. The subdeltoid bursa was excised to visualize the supraspinatus tendon. A longitudinal incision was made $1.5 \mathrm{~cm}$ posterior to the biceps muscle near the greater tuberosity to which the supraspinatus muscle attaches to expose the insertion point of the nail. A drill and awl were used to create an insertion point at $1.5 \mathrm{~cm}$ posterior to the bicipital groove and the border between the joint surface and greater tuberosity. A nail was then inserted through the medial sulcus of the greater tuberosity to obtain intramedullary access. To prevent subacromial impingement by nail protrusion, the proximal end of the nail was inserted within the articular margin of the humeral head. We did not damage the deltoid branch of the axillary nerve. For complex fractures, two Steinmann pins were inserted into the proximal and distal humeral shaft based on the fracture line, then adjusted to reduce the alignment and rotation of the humeral shaft while viewing the fluoroscopic image. To prevent radial nerve injury, a $2 \mathrm{~cm}$ longitudinal incision was conducted in nine cases, and distal screws were inserted after visualizing the pathway of the radial nerve.

\section{Postoperative Rehabilitation Treatment}

All patients wore an abduction brace for 1 month after the operation, and an arm sling was worn for another 1 month after removing the abduction brace. Pendulum, passive elevation and isometric exercises were performed on the first postoperative day when the fixation was firm. At the 1 month follow-up, an 
active elevation exercise with $90^{\circ}$ forward flexion of the glenohumeral joint was performed. At the 6 to 8 weeks follow-up, active exercise was performed according to clinical union time. During the follow-up period, the active exercise was stopped when the migration of proximal screws occurred. After observing pain relief at the surgical site and the degree of screw migration, a decision of whether to resume the exercise was made.

\section{Statistical Analysis}

A paired t-test was used to compare the results between groups, including radiological and clinical union, operation time, and KSS. IBM SPSS ver. 23.0 (IBM Corp., Armonk, NY, USA) was utilized for statistical analysis, and a $p<0.05$ indicated statistical significance.

\section{Ethical Approval}

Institutional review board approval (No. CR-18-128-L) was obtained and informed consent was acquired from all individual patients included in this research. Additionally, all participants of this study agreed to its publication, including that of the attached radiographic images.

\section{Results}

All cases progressed to union, resulting in a 100\% radiological union rate. Radiological union was confirmed by both plain AP and lateral radiographs at an average of 5.0 months in group I and 8.4 months in group II, respectively. The difference between the two groups was statistically significant $(p<0.01)$. Clinical union was observed at 1.6 months in group I and 2.0 months in group II, but there was no significant difference between groups $(p=0.441)$. Operative time was recorded as 83.6 in group I, and 101.6 minutes in group II, with significant differences between the two groups $(p<0.01)$. The mean KSS scores were 89.7 and 90.6, but these did not differ significantly between groups $(p=0.352)$. The mean postoperative visual analogue scale scores were 0.6 and 0.8 , which also did not differ significantly difference $(p=0.493)$ (Table 2). Overall, no blood transfusion was required after the surgery. In terms of complications, one case of radial nerve palsy occurred. For this case, a wrist and thumb extension of motor grade 2 were observed on the first day after the surgery. The partial tear of the radial nerve was confirmed in the operating room immediately after the palsy, and this was recovered at 6 months after neurorrhaphy. The remaining complications were minor, including two cases of subacromial impingement because of nail protrusion (6.6\%) and five cases of migration of proximal cancellous screws (16.6\%). However, none of these minor complications affected the union time and outcome. Subacromial impingement because of nail protrusion required nail removal, and migration of proximal cancellous screws was followed by removal of the protruded screws.
Table 2. Comparison of Radiographic and Clinical Outcome between Group I and II

\begin{tabular}{lccc}
\hline \multicolumn{1}{c}{ Variable } & Group I $(\mathrm{n}=15)^{*}$ & Group II $(\mathrm{n}=15)^{*}$ & $p$-value \\
\hline Radiological union (mo) & $5.0 \pm 1.41$ & $8.4 \pm 1.81$ & $<0.01$ \\
Clinical union (mo) & $1.6 \pm 0.66$ & $2.0 \pm 0.61$ & 0.441 \\
KSS & $89.7 \pm 6.01$ & $90.6 \pm 5.72$ & 0.352 \\
Operation time (min) & $83.6 \pm 10.21$ & $101.6 \pm 10.52$ & $<0.01$ \\
Postoperative VAS & $0.6 \pm 0.12$ & $0.8 \pm 0.25$ & 0.493 \\
\hline
\end{tabular}

Values are presented as mean \pm standard deviation.

KSS: Korean shoulder scoring system, VAS: visual analogue scale.

${ }^{*}$ Group I was type A (simple) and B (wedge), Group II was type C (complex fractures); each types were based on the Orthopaedic Trauma Association/Arbeitsgemeinschaft für Osteosynthesefragen fracture classification system.

\section{Discussion}

Nonoperative management is the main treatment method for humeral shaft fracture, and many reports have shown good clinical results after nonoperative treatment for humeral shaft fracture. $^{6-8)}$ However, in many cases, the fracture cannot be resolved with nonoperative management. Operative indications for humeral shaft fracture generally include polytrauma, open fractures, pathological fractures, vascular injury requiring repair, and brachial plexus injury. In addition, surgery is indicated even if acceptable alignment is not maintained with a functional brace. Klenerman" reported that a $30^{\circ}$ varus or $20^{\circ}$ anterior angulation were the limits for no risk of deformation, and these values have been considered as radiographic parameters for nonoperative treatment. Furthermore, Shields et al. ${ }^{10)}$ reported that a valgus deformity less than $30^{\circ}$, rotational deformity below $15^{\circ}$, and shortening of less than $3 \mathrm{~cm}$ were acceptable. In the present study, surgery was performed in patients with varus angulation greater than $30^{\circ}$ or anterior angulation of more than $20^{\circ}$.

Several options for operative management in humeral shaft fracture can be considered because of advances in modalities for internal fixation such as plates, intramedullary nails or external fixation. Each modality can be selected according to various factors such as surgeon preference, fracture pattern, possibility of radial nerve damage, and soft tissue condition, ${ }^{1)}$ but the most widely preferred method is open reduction and internal fixation with plate-and-screw constructs. However, although the internal fixation with plates has historically been regarded as the golden standard of surgical management for humeral shaft fracture, no single surgical technique or device has been shown to be much better than any other. Zhao et al. ${ }^{11)}$ reported that there was no significant difference in union rate, infection, or radial nerve injury between intramedullary nailing and plate fixation for humeral shaft fracture, Hohmann et al. ${ }^{12)}$ reported that minimally invasive plating showed better outcomes than intramedullary nailing or open reduction and plate fixation. We preferred in- 
tramedullary nailing for humeral shaft fracture because it had several advantages. Specifically, the intramedullary nail is located near the mechanical axis of humeral shaft, which can reduce bending loads and potentially mitigate stress shielding. ${ }^{13,14)}$ Second, although there is controversy about rotator cuff injury, a small surgical incision can be made to decrease soft tissue injury as well as preserve the periosteal blood supply, which may have a positive effect on the bone union. Radial nerve palsy may occur during reduction and insertion of a guide wire or distal locking screws, but many studies have reported that the incidence of radial nerve palsy is comparable to plate fixation. ${ }^{11,15-17)}$

The most widely used classification in humeral shaft fractures is the OTA/AO combined classification system. ${ }^{18)}$ In this system, the humeral shaft fractures are mainly categorized into three types: simple (type A), wedge (type B), and complex (type C) fractures. Each type is further classified according to the fracture pattern. In this study, we divided the patients into two groups: group I (type A and B) and group II (type C). The reason we classified the groups as above was because of the difficulty at which we can restore the original length of the humeral shaft by inducing cortical bone contact during the reduction and nail insertion procedure. Intramedullary nailing does not require perfect contacts between bone fragments to obtain relative stability. However, in our experiences, the restoration of the upper arm length after surgery could greatly affect the recovery period of postoperative activities and range of motion, and the period of radiological union differs according to fracture types. The radiological union period in this study showed statistically significant differences between the two groups. The reason for the difference in operation time between the two groups is thought to have been caused by the difference in time required to restore the original length of the upper extremity to the greatest degree possible.

Several studies regarding management of humeral shaft fractures with intramedullary nailing have raised various complications. Issues pertaining to intramedullary nailing include insertion site morbidity, subacromial impingement and reoperation rate. ${ }^{19,20)}$ The cause of these issues are believed to be either subacromial impingement, which can occur in response to a prominent nail or injury to the rotator cuff. Many surgeons have suggested different approaches that include avoidance of the avascular zone of the rotator cuff and careful repair of the tendon with improved outcomes. ${ }^{20-23)}$ In the present study, the supraspinatus was carefully repaired after nail fixation, and no rotator cuff re-tears were found on follow-up. To prevent subacromial impingement by nail protrusion, the proximal end of the nail was inserted within the articular margin of the humeral head; however, there were two cases of subacromial impingement during follow-up period.

Radial nerve injury, which occurs in up to $18 \%$ of closed injuries ${ }^{24)}$ is also a common complication that is related to middle one-third spiral humeral shaft fractures. ${ }^{25)}$ Based on observations after 4 months of follow-up, there is an expected recovery rate of $90 \%{ }^{24)}$ Surgical exploration is not necessary except in cases of open fracture, penetrating injury, nerve palsy after closed reduction, or when there is no recovery at 4 months after the injury. When the distal screws were inserted, we did not check the pathway of the radial nerve through visual confirmation. However, radial nerve palsy occurred in July 2015, and the radial nerve pathway was visually confirmed in all cases after that.

The loosening of proximal cancellous screws reportedly occurs in $4 \%$ to $20 \%$ of cases. ${ }^{26,27)}$ In such cases, it is possible that the Polarus intramedullary nail failed to hold the screws because it did not have a locking mechanism. To prevent this complication, Inoue et al. ${ }^{28}$ suggested that the second and third proximal screws be positioned by penetrating into the far cortex. However, to prevent loosening, the injury of the articular cartilage in the humeral head may cause osteoarthritis. For these reasons, we inserted 3 or 4 proximal screws without passing through the far cortex. When screw loosening of more than $3 \mathrm{~mm}$ occurred, it was removed. In our study, the migration of proximal cancellous screws occurred in five cases; however, it did not affect the union time or outcome.

It should be noted that there are several limitations to this study. Specifically, it is difficult to generalize the results of the research because only 30 cases were considered. In addition, it was also a retrospective comparative study, and the patient assignments were not randomized. Moreover, we included a wide range of patients from 20 to 87 years old, and did not consider bone union time and rate according to age. Finally, to illustrate the superiority of intramedullary nailing, it must be compared with other surgical techniques.

\section{Conclusion}

Radiologic union and good clinical outcome were obtained in all cases in this study, regardless of fracture type. In cases of the complex type, although it took more time than radiological union, there were no differences in the clinical results compared to the simple and wedge type unions. Intramedullary nailing using the Polarus humeral nail is considered to be a good treatment modality for all types of humeral shaft fractures, especially for the treatment of complex types of injuries, such as segmental or comminuted humeral shaft fractures.

\section{References}

1. Updegrove GF, Mourad W, Abboud JA. Humeral shaft fractures. J Shoulder Elbow Surg. 2018;27(4):e87-97. doi: 10.1016/j.jse.2017.10.028.

2. Verdano MA, Pellegrini A, Schiavi P, Somenzi L, Concari G, Ceccarelli F. Humeral shaft fractures treated with antegrade intramedullary nailing: what are the consequences for the 
rotator cuff? Int Orthop. 2013;37(10):2001-7. doi: 10.1007/ s00264-013-2007-1.

3. Walker M, Palumbo B, Badman B, Brooks J, Van Gelderen J, Mighell M. Humeral shaft fractures: a review. J Shoulder Elbow Surg. 2011;20(5):833-44. doi: 10.1016/j.jse.2010.11.030.

4. Koike Y, Komatsuda T, Sato K. Internal fixation of proximal humeral fractures with a polarus humeral nail. J Orthop Traumatol. 2008;9(3):135-9. doi: 10.1007/s10195-008-0019-1.

5. Kazakos K, Lyras DN, Galanis V, et al. Internal fixation of proximal humerus fractures using the polarus intramedullary nail. Arch Orthop Trauma Surg. 2007;127(7):503-8. doi: 10.1007/ s00402-007-0390-z.

6. Ali E, Griffiths D, Obi N, Tytherleigh-Strong G, Van Rensburg L. Nonoperative treatment of humeral shaft fractures revisited. J Shoulder Elbow Surg. 2015;24(2):210-4. doi: 10.1016/ j.jse.2014.05.009.

7. Rutgers M, Ring D. Treatment of diaphyseal fractures of the humerus using a functional brace. J Orthop Trauma. 2006;20(9):597-601. doi: 10.1097/01.bot.0000249423. 48074.82.

8. Sarmiento A, Zagorski JB, Zych GA, Latta LL, Capps CA. Functional bracing for the treatment of fractures of the humeral diaphysis. J Bone Joint Surg Am. 2000;82(4):478-86.

9. Klenerman L. Fractures of the shaft of the humerus. J Bone Joint Surg Br. 1966;48(1):105-11.

10. Shields E, Sundem L, Childs S, et al. The impact of residual angulation on patient reported functional outcome scores after non-operative treatment for humeral shaft fractures. Injury. 2016;47(4):914-8. doi: 10.1016/j.injury.2015.12.014.

11. Zhao JG, Wang J, Wang C, Kan SL. Intramedullary nail versus plate fixation for humeral shaft fractures: a systematic review of overlapping meta-analyses. Medicine (Baltimore). 2015;94(11):e599. doi: 10.1097/MD.0000000000000599.

12. Hohmann E, Glatt V, Tetsworth K. Minimally invasive plating versus either open reduction and plate fixation or intramedullary nailing of humeral shaft fractures: a systematic review and meta-analysis of randomized controlled trials. J Shoulder Elbow Surg. 2016;25(10):1634-42. doi: 10.1016/j.jse.2016.05.014.

13. Korompilias AV, Lykissas MG, Kostas-Agnantis IP, Vekris MD, Soucacos PN, Beris AE. Approach to radial nerve palsy caused by humerus shaft fracture: is primary exploration necessary? Injury. 2013;44(3):323-6. doi: 10.1016/j.injury.2013.01.004.

14. Gerwin M, Hotchkiss RN, Weiland AJ. Alternative operative exposures of the posterior aspect of the humeral diaphysis with reference to the radial nerve. J Bone Joint Surg Am. 1996;78(11):1690-5.

15. Gottschalk MB, Carpenter W, Hiza E, Reisman W, Roberson J. Humeral shaft fracture fixation: incidence rates and complications as reported by American Board of Orthopaedic Surgery part II candidates. J Bone Joint Surg Am. 2016;98(17):e71. doi: 10.2106/JBJS.15.01049.
16. Kurup H, Hossain M, Andrew JG. Dynamic compression plating versus locked intramedullary nailing for humeral shaft fractures in adults. Cochrane Database Syst Rev. 2011;(6):CD005959. doi: 10.1002/14651858.CD005959.pub2.

17. Ouyang H, Xiong J, Xiang P, Cui Z, Chen L, Yu B. Plate versus intramedullary nail fixation in the treatment of humeral shaft fractures: an updated meta-analysis. J Shoulder Elbow Surg. 2013;22(3):387-95. doi: 10.1016/j.jse.2012.06.007.

18. Marsh JL, Slongo TF, Agel J, et al. Fracture and dislocation classification compendium - 2007: Orthopaedic Trauma Association classification, database and outcomes committee. J Orthop Trauma. 2007;21(10 Suppl):S1-133.

19. Changulani M, Jain UK, Keswani T. Comparison of the use of the humerus intramedullary nail and dynamic compression plate for the management of diaphyseal fractures of the humerus. A randomised controlled study. Int Orthop. 2007;31(3):391-5. doi: 10.1007/s00264-006-0200-1.

20. Rommens PM, Kuechle R, Bord T, Lewens T, Engelmann R, Blum J. Humeral nailing revisited. Injury. 2008;39(12):131928. doi: 10.1016/j.injury.2008.01.014.

21. Dimakopoulos P, Papadopoulos AX, Papas M, Panagopoulos A, Lambiris E. Modified extra rotator-cuff entry point in antegrade humeral nailing. Arch Orthop Trauma Surg. 2005;125(1):2732. doi: 10.1007/s00402-004-0757-3.

22. Park JY, Pandher DS, Chun JY, Lee ST. Antegrade humeral nailing through the rotator cuff interval: a new entry portal. J Orthop Trauma. 2008;22(6):419-25. doi: 10.1097/BOT. Ob013e318173f751.

23. Christ AB, Gausden EB, Warner SJ, Nellestein AM, Thacher RR, Lorich DG. Rotator cuff-sparing approach for antegrade humeral nailing with biceps tenodesis: a technical trick with clinical implications. J Orthop Trauma. 2017;31(2):e60-5. doi: 10.1097/BOT.0000000000000684.

24. Pollock FH, Drake D, Bovill EG, Day L, Trafton PG. Treatment of radial neuropathy associated with fractures of the humerus. J Bone Joint Surg Am. 1981;63:239-43.

25. Shao YC, Harwood P, Grotz MR, Limb D, Giannoudis PV. Radial nerve palsy associated with fractures of the shaft of the humerus: a systematic review. J Bone Joint Surg Br. 2005;87(12):164752. doi: 10.1302/0301-620X.87B12.16132.

26. Agel J, Jones CB, Sanzone AG, Camuso M, Henley MB. Treatment of proximal humeral fractures with polarus nail fixation. J Shoulder Elbow Surg. 2004;13(2):191-5. doi: 10.1016/ S1058274603003100.

27. Rajasekhar C, Ray PS, Bhamra MS. Fixation of proximal humeral fractures with the polarus nail. J Shoulder Elbow Surg. 2001;10(1):7-10. doi: 10.1067/mse.2001.109556.

28. Inoue H, Sato K, Ito K, Inawashiro T. [Internal fixation of proximal humeral fractures with a Polarus humeral nail]. Bessatsu Seikeigeka. 2003;44:23-7. Japanese. 